\title{
Toward Privacy Enhanced Solutions For Granular Control Over Health Data Collected by Wearable Devices
}

\author{
Byron M. Lowens \\ Clemson University \\ blowens@clemson.edu
}

\begin{abstract}
The advent of wearable technologies has engendered novel ways to understand human behavior as it relates to personalized healthcare and health management. As the availability of these technologies expand and proliferate among users, concerns about threats to data privacy have been raised, specifically, regarding the collection and dissemination of data from wearable devices. These factors point to the urgency to better understand user sharing preferences to formulate personalized solutions that give users granular control of the data collected by their wearable devices. The goal of my dissertation is to design and build human-centered solutions that address the need for granular privacy control over data generated by wearable devices.
\end{abstract}

\section{CCS CONCEPTS}

- Security and privacy $\rightarrow$ Usability in security and privacy; • Human-centered computing $\rightarrow$ Empirical studies in HCI;

\section{KEYWORDS}

Privacy; Wearables; Gesture Elicitation; HCI

\section{ACM Reference Format:}

Byron M. Lowens. 2018. Toward Privacy Enhanced Solutions For Granular Control Over Health Data Collected by Wearable Devices. In MobiSys PhD Forum'18: , June 10, 2018, Munich, Germany. ACM, New York, NY, USA, 2 pages. https://doi.org/10.1145/3212711.3212714

\section{INTRODUCTION}

User-generated data from wearable devices is engendering significant advantages for users of these devices and a myriad of societal benefits [15]. Wearable devices provide innovative methods for monitoring and potentially enhancing users' health and well-being $[4,11,17]$. Each device, irrespective of its form factor, achieves one or more limited set of tasks: dynamically recording and streaming biometric data, recording data for later collection or providing real-time feedback to the user [3].

While these types of technologies are increasingly available to users, managing information privacy with those technologies is becoming more difficult, as more data are collected and exchanged $[1,13]$. Prior work cites privacy as a key user concern for adopting

Permission to make digital or hard copies of part or all of this work for personal or classroom use is granted without fee provided that copies are not made or distributed for profit or commercial advantage and that copies bear this notice and the full citation on the first page. Copyrights for third-party components of this work must be honored.

For all other uses, contact the owner/author(s).

MobiSys PhD Forum'18, June 10, 2018, Munich, Germany

(C) 2018 Copyright held by the owner/author(s).

ACM ISBN 978-1-4503-5841-5/18/06.

https://doi.org/10.1145/3212711.3212714 pervasive computing technologies[16, 19], more specifically interactive systems in areas of healthcare,personal communications [8], and wearable computing $[9,12,13]$. These concerns are expected to continue to grow because very little is known about wearable privacy from an $\mathrm{HCI}$ perspective $[7,13,18]$.

As wearable technologies become more complex, the way data are collected and used from these devices becomes more opaque. The lack of transparency could lead to instances where data generated by wearable devices are revealed without user consent, or used inappropriately, which in turn could present severe consequences for users, [6] and eventually become, what we recognize to be a "skeleton in the closet [10]." Each person who uses these types of technologies has an activity history that justly belongs to that individual, and should not be used to surveil, limit, or coerce the person who generated the data by the wearable device.

In addition to the concerns that scientific studies have raised and that I briefly outline, further exploration of wearable privacy from an HCI perspective, is also a question of extreme practical importance. For example, in a 2018 article published by Mobi Health News [14] Fitbit medical director John Moore said, 'We need to really invest in privacy and security, and also be incredibly transparent with people about what's being shared and with whom, so they can make the right decisions about what they want to do with their data."

Taken together these concerns suggest a need to protect user privacy by implementing privacy-enhanced solutions to give users granular control over their information.

\section{GOAL AND MOTIVATION}

Prior work [2] reveals that users desire granular control over the sharing their health-related information; users only want to share certain parts of their data with specific recipients, while restricting other parts of their data. The same work also maintains that, for users to have meaningful privacy control over their health related data, granular control over personal information is essential. Additionally, users are concerned about the unintended use of data collected by wearable devices and desire more control data collected by wearable devices [10]. These key aspects are the motivations behind my doctoral work.

The overarching goal of my dissertation research is to design technologies that give users more granular control over the health related data collected by wearables. Affording granular control to the user, gives them ordnance over the collection, processing, and sharing of their personal health information, while removing concerns related to the unintended usage of their personal wearable data $[5,10]$.

To that end, I have started and will continue to conduct studies which serve as the basis for my dissertation. A scenario-based 
experiment including a gesture elicitation component was conducted, with the goal of understanding user's granular privacy and sharing preferences for the data collected by wearable devices. Additionally, we sought to understand what gestures would users elicit to imply their sharing preferences of that data. In this study, gestures were elicited as the articulation of choice. Gestures have long been explored as an interaction method through the use of technology[20, 21]. More importantly, the decision to elicit gestural interaction is based on the notion that users should able to express contextual privacy decisions during data collection which makes the gestural component of the study important.

In the near future, my plan is to conduct a cross-sectional survey to evaluate the acceptability of this user-defined gesture set, to develop a taxonomy of gestures that give users' granular control over their information.

From this work, we seek to answer the following research questions: (i) Are users sharing preferences associated with: the type of data being shared, recipient of the data, sensitivity of the data, valence of the data and participant views on privacy?; (ii) What type of gestures are used in privacy intensive scenarios for wearable devices?; (iii) How do gestures differ depending on who is around and who the data is being shared with?

Identifying and understanding these factors will allow us to identify unexplored design alternatives that enable granular privacy control on wearable devices.

\section{METHODOLOGICAL APPROACH}

The initial study employed a scenario-based fractional factorial mixed design experiment,in which user granular and privacy sharing preferences for wearable devices were explored. The purpose of study was to understand user preferences for sharing health-related information from a wearable device (WWD/HMD) using a given set of parameters. Within this scenario-based experiment, a gesture elicitation study investigated which user-defined gestures participants elicited to indicate their sharing preference with specific recipients. During this experimentl, participants demonstrated the gesture they would use to convey their sharing preference.

In the study, 32 participants received a set of 12 scenarios. For each scenario, participants imagined receiving a notification regarding the type of health-related data that was collected and asked if they would share this information with 1 of 4 recipients. Once participants indicated their sharing preference, they were asked perform a gesture based on this response. Participants also rated their level of sensitivity for the given data in the scenario using a 4-point Likert scale, ranging from very sensitive to not at all sensitive and also rated the valence of the data on a 5 t scale ranging from "Very Negative" to "Very Positive."

\section{EXPECTED CONTRIBUTIONS}

The primary contribution of this work includes:

- A more thorough understanding of factors that influence users' intentions to share health data from a wearable devices.

- A quantitative and qualitative characterization of user-defined privacy gestures for wrist-worn and head-mounted wearable devices.
- A working prototype system that enables users to control privacy at a granular level using gestures.

- An evaluation of the effectiveness of the system at improving privacy control.

Together these studies will provide insight into users' wearable data sharing preferences and allow us to develop novel interaction methods that afford granular privacy control for user data generated by wearable device.

\section{ACKNOWELDGMENTS}

This material is based upon work supported by the National Science Foundation under Grant No. 1619950, 1565268 and 1314342. I would also like to thank Dr. Kelly Caine for helping formulate the dissertation questions and methodological approach, Dr. Bart Knijnenburg for guidance about statistical analysis, and the entire Amulet Team (amulet-project.org) for input and feedback.

\section{REFERENCES}

[1] Syagnik Banerjee, Thomas Hemphill, and Phil Longstreet. 2018. Wearable devices and healthcare: Data sharing and privacy. The Information Society 34, 1 (2018).

[2] Kelly Caine and Rima Hanania. 2013. Patients want granular privacy control over health information in electronic medical records. Fournal of the American Medical Informatics Association 20, 1 (2013).

[3] Peter R Chai, Roger Y Wu, Megan L Ranney, Paul S Porter, Kavita M Babu, and Edward W Boyer. 2014. The virtual toxicology service: wearable head-mounted devices for medical toxicology. Journal of Medical Toxicology 10, 4 (2014).

[4] Bruce H Dobkin. 2013. Wearable motion sensors to continuously measure realworld physical activities. Current opinion in neurology 26, 6 (2013).

[5] Vivian Genaro Motti and Kelly Caine. 2015. An overview of wearable applications for healthcare: requirements and challenges. In UbiComp/ISWC'15 Adjunct. ACM.

[6] Cory Hallam and Gianluca Zanella. 2016. Wearable Device Data and Privacy: A study of Perception and Behavior. World 7, 1 (2016).

[7] Roberto Hoyle, Robert Templeman, Steven Armes, Denise Anthony, David Crandall, and Apu Kapadia. 2014. Privacy behaviors of lifeloggers using wearable cameras. In Ubicomp '14. ACM.

[8] Giovanni Iachello, Jason Hong, et al. 2007. End-user privacy in human-computer interaction. Foundations and Trends ${ }^{\circledR}$ in Human-Computer Interaction 1 (2007).

[9] Linda Lee, J Lee, Serge Egelman, and David Wagner. 2016. Information disclosure concerns in the age of wearable computing. In NDSS Workshop on Usable Security (USEC), Vol. 1.

[10] Byron Lowens, Vivian Genaro Motti, and Kelly Caine. 2017. Wearable Privacy: Skeletons in The Data Closet. In Healthcare Informatics (ICHI), 2017 IEEE International Conference on. IEEE.

[11] Hawley E Montgomery-Downs, Salvatore P Insana, and Jonathan A Bond. 2012. Movement toward a novel activity monitoring device. Sleep and Breathing 16, 3 (2012).

[12] Vivian Genaro Motti and Kelly Caine. 2014. Understanding the wearability of head-mounted devices from a human-centered perspective. In Proceedings of the 2014 ACM International Symposium on Wearable Computers. ACM.

[13] Vivian Genaro Motti and Kelly Caine. 2015. Users' privacy concerns about wearables. In International Conference on Financial Cryptography and Data Security. Springer.

[14] Dave Muoio. 2018. Fitbit, UnitedHealth, Empatica heads discuss data privacy challenges for wearables. http://www.mobihealthnews.com/content/ fitbit-unitedhealth-empatica-heads-discuss-data-privacy-challenges-wearables

[15] Future of Privacy Forum. 2016. https://fpf.org/wp-content/uploads/2016/08/ FPF-Best-Practices-for-Wearables-and-Wellness-Apps-and-Devices-Final.pdf.

[16] Thad Starner. 2001. The challenges of wearable computing: Part 2. Ieee Micro 21 (2001).

[17] Matteo Stoppa and Alessandro Chiolerio. 2014. Wearable electronics and smart textiles: a critical review. Sensors 14, 7 (2014).

[18] Emily Troshynski, Charlotte Lee, and Paul Dourish. 2008. Accountabilities of presence. In CHI 2008.

[19] Roy Want, Bill N Schilit, Norman I Adams, Rich Gold, Karin Petersen, David Goldberg, John R Ellis, and Mark Weiser. 1995. An overview of the PARCTAB ubiquitous computing experiment. IEEE personal communications 2, 6 (1995)

[20] Daniel Wigdor and Dennis Wixon. 2011. Brave NUI world: designing natural user interfaces for touch and gesture. Elsevier.

[21] Jacob O Wobbrock, Meredith Ringel Morris, and Andrew D Wilson. 2009. Userdefined gestures for surface computing. In CHI 2009. ACM. 